\title{
Reviewer acknowledgement 2014
}

Koji Tsuboi

\section{Contributing reviewers}

The BioPsychoSocial Medicine editorial team would like to thank the following colleagues who contributed to peer review for the journal in Volume 8 (2014).

Mahmoud AL-Omiri
Jordan
Marie Amitani
Japan
Tetsuya Ando
Japan
Shuji Aou
Japan
Akihiro Asakawa
Japan
Takanobu Baba
Japan
Vassilis Barkoukis
Greece
Ananda Balayogi Bhavanani
India
Bruno Bonaz
France
Isabelle Bray
United Kingdom
Marc Cohen
Australia
Yukihiko Fujita
Japan
Shin Fukudo
Japan
Mikihiko Fukunaga
Japan

Toyohiro Hamaguchi
Japan
Makoto Hashiro
Japan
Makoto Hashizume
Japan
Peter Herschbach
Germany
Taina Hintsa
Finland
Reiko Hori
Japan
Bjoern Horing
United States of America
Mizuho Hosogi
Japan
Lai Ming Christy Hui
Hong Kong
Akio Inui
Japan
Yuko Ishizaki
Japan
Tsutomu Kamei
Japan
Kenji Kanbara
Japan
Michiko Kano
Japan

\author{
Norito Katoh \\ Japan \\ Keisuke Kawai \\ Japan

\section{Kenji Kawakita} \\ Japan \\ Monika Keller \\ Germany \\ Hiroe Kikuchi \\ Japan
Masayo Kojima
Japan

Hiroaki Kumano

Japan

Hubert Lacey

United Kingdom

Richard Lane

United States of America

Ichiro Mashima

Japan

Jason Mazanov

Australia

Christian Montag

Germany

Astrid Müller

Germany

Takao Munemoto

Japan

Correspondence: tsuboic@med.toho-u.ac.jp

Department of Psychosomatic Medicine, Toho University School of Medicine,

Tokyo, Japan 
Tomohiko Muratsubaki

Japan

Yoshikatsu Nakai

Japan

Mutsuhiro Nakao

Japan

Nobuo Nishi

Japan

Shinobu Nomura

Japan

Takehiro Nozaki

Japan

Yuko Odagiri

Japan

Daisuke Ohta

Japan

Takakazu Oka

Japan
Gaby Resmark

Germany

Annelieke Roest

Netherlands

Hironori Shimada

Japan

Tomotaka Shoji

Japan

Katsuhisa Sunada

Japan

Shu Takakura

Japan

Takeaki Takeuchi

Japan

Yoshiyuki Takimoto

Japan

Hidetaka Tanaka

Japan
Jun Tayama

Japan

Yuri Terasawa

Japan

Daniel Widmer

Switzerland

Jong Min Woo

Korea, South

Jumpei Yajima

Japan

Kosuke Yamada

Japan

Kazufumi Yoshihara

Japan

Kazuhiro Yoshiuchi

Japan

Stephan Zipfel

Germany 